\title{
Estimates of Kato-Temple Type for n-dimensional Spectral Measures
}

\author{
By
}

\author{
S. T. KURODA*
}

\begin{abstract}
The Kato-Temple estimate for an isolated eigenvalue of a selfadjoint operator is extended to spectral measures on $\mathbf{R}^{n}$, or equivalently, a commuting set of $n$ selfadjoint operators. The proof depends on a general variational characterization of the spectrum. In the case of normal operators this corresponds to Rayleigh bounds applied to resolvents. It is also shown that the obtained estimate has a certain invariance property under an inversion of the space.
\end{abstract}

\section{$\S 1 . \quad$ Introduction and Main Result}

\section{§1.1. Introduction}

Let $A=\int_{-\infty}^{\infty} \lambda d E(\lambda)$ be a selfadjoint operator in a Hilbert $\mathcal{H}$ with the domain $\mathcal{D}(A)$ and the spectrum $\sigma(A)$. For $u \in \mathcal{D}(A)$ with $u \neq 0$ we put

$$
\eta=\eta(u ; A)=(A u, u) /\|u\|^{2}, \quad \varepsilon=\varepsilon(u ; A)=\|(A-\eta I) u\| /\|u\| .
$$

$\eta$ is called the Rayleigh quotient and $\varepsilon$ is the norm of the residual after normalization. When $u$ is an approximate eigenvector of an isolated eigenvalue $\lambda_{0}$ of $A$, the Rayleigh quotient is expected to be an approximation of the eigenvalue $\lambda_{0}$ with an error estimate of the form $\left|\eta-\lambda_{0}\right| \leq$ const $\cdot \varepsilon^{2}$. In order to obtain a precise estimate of this type it is often required to have a rough a priori information on the location of $\sigma(A)$. The following classical result of $\mathrm{G}$. Temple and T. Kato ([8], [4]) is known as the Kato-Temple inequality (cf. [2]).

Communicated by T. Kawai. Received August 7, 2006. Revised October 30, 2006.

2000 Mathematics Subject Classification(s): Primary 47P10 49R50; Secondary 35P15 $65 \mathrm{~F} 15$.

*3-7-5-604 Ebisuminami, Shibuya-ku, Tokyo 150-0022, Japan.

e-mail: kuroda-s@khaki.plala.or.jp 
Theorem A. Let $A$ be a selfadjoint operator and let $-\infty<\alpha<\beta<\infty$. Assume that $(\alpha, \beta) \cap \sigma(A)$ consists of at most one point. Let $u \in \mathcal{D}(A)$ and let $\eta$ and $\epsilon$ be as in (1.1). If $\varepsilon^{2}<(\eta-\alpha)(\beta-\eta)$, then $(\alpha, \beta)$ consists of exactly one point, i.e., $(\alpha, \beta) \cap \sigma(A)=\left\{\lambda_{0}\right\}$, and $\lambda_{0}$ is bounded as

$$
\lambda_{-} \equiv \eta-\frac{\epsilon^{2}}{\beta-\eta} \leq \lambda_{0} \leq \eta+\frac{\epsilon^{2}}{\eta-\alpha} \equiv \lambda_{+} .
$$

Note that the assumption $\varepsilon^{2}<(\eta-\alpha)(\beta-\eta)$ implies in particular that $\eta \in(\alpha, \beta)$. A feature of this theorem is that $\eta$ is not necessarily the center of the interval $\left(\lambda_{-}, \lambda_{+}\right)$.

The purpose of the present paper is to prove a similar theorem for an $n$-dimensional spectral measure, or equivalently, for a commuting family of $n$ selfadjoint operators. For this purpose it is convenient to rewrite (1.2) in terms of centers and half length of intervals. Put

$$
a=(\alpha+\beta) / 2, \quad r=(\beta-\alpha) / 2 .
$$

Then, by a simple manipulation we see that estimate (1.2) is equivalent to

$$
\left|\lambda_{0}-\left(\eta-\frac{\varepsilon^{2}}{r^{2}-|\eta-a|^{2}}(\eta-a)\right)\right| \leq \frac{r \varepsilon^{2}}{r^{2}-|\eta-a|^{2}} .
$$

We shall show that in this form Theorem A can be generalized to $n$-dimensional spectral measures (see Theorem 1.1). There the interval $(\alpha, \beta)$ is replaced by an open ball $B(a ; r)$ and, instead of the interval $\left(\lambda_{-}, \lambda_{+}\right)$, a ball $B_{0}$ will be obtained as a ball enclosing the spectrum in $B(a ; r)$. In addition, the assumption in Theorem A that $(\alpha, \beta) \cap \sigma(A)$ consists of at most one point will be slightly generalized with a corresponding modification of the conclusion.

With $n=2$ Theorem 1.1 gives a generalization of Theorem A to normal operators. For normal operators E. M. Harrell ([3]) partly generalized Theorem A, but the obtained disk for estimating $\lambda_{0}$ is a disk with the center $\eta$. (For normal matrices a similar result is in $[9$, p. 188].)

The rest of the present paper is organized as follows. In $\S 1.2$ we shall make a brief review of $n$-dimensional spectral measures partly for fixing notations. Main theorem (Theorem 1.1) is presented in $\S 1.3$ and is proved in $\S 2$. In $\S 2.1$ a generalized form of the variational Rayleigh bound is presented (Lemma 2.1). In $\S 2.2$, by applying this lemma to a mapping of inversion in $\mathbf{R}^{n}$, we construct a family of balls $B(\omega)$ with a parameter $\omega,|\omega|=1$, which contains the part of the spectrum we are concerned with. Relations between $B(\omega)$ and $B_{0}$ will be exploited in $\S 2.3$ by elementary computations (Proposition 2.3 ). In short, $B_{0}$ 
is shown to be the intersection of all $B(\omega)$. The last part of the proof in $\S 2.4$ is a simple geometric argument. Finally, in $\S 3$ an invariance property which $B_{0}$ enjoys under a mapping of inversion will be discussed.

Throughout the present paper we use the following notations. $B(a ; r)$ and $S(a ; r)$ denote the open ball and the sphere, respectively, with the center $a \in \mathbf{R}^{n}$ and the radius $r>0: B(a ; r)=\left\{x \in \mathbf{R}^{n}|| x-a \mid<r\right\}, S(a ; r)=$ $\left\{x \in \mathbf{R}^{n}|| x-a \mid=r\right\}$. The closure of a set $B$ is denoted by $\bar{B}$. For $B(a ; r)$ we write $\bar{B}(a ; r)$ instead of $\overline{B(a ; r)} \cdot \operatorname{diam}(A)=\sup \{|x-y| \mid x, y \in A\}$ denotes the diameter of a set $A \subset \mathbf{R}^{n}$. For convenience we agree that $\operatorname{diam}(\emptyset)=0$ where $\emptyset$ denotes the empty set. Accordingly $\operatorname{diam}(A)=0$ means that either $A$ consists of one point or $A$ is empty.

We denote by $\mathcal{D}(T)$ the domain of an operator $T$.

\section{§1.2. Spectral measures on $\mathbf{R}^{n}$}

Let $\mathcal{H}$ be a Hilbert space and let $\mathcal{P}$ be the set of all orthogonal projections in $\mathcal{H}$. Let $\mathcal{B}$ be the family of all Borel sets in $\mathbf{R}^{n}$. A mapping $E$ from $\mathcal{B}$ to $\mathcal{P}$ is called a spectral measure on $\mathbf{R}^{n}$ if $E$ is strongly $\sigma$-additive and $E\left(\mathbf{R}^{n}\right)=I$. For any $u, v \in \mathcal{H},(E(\cdot) u, v)$ is a complex valued measure on $\mathbf{R}^{n}$. In particular, $(E(\cdot) u, u) \geq 0$ is a measure. (For general spectral measures see [1], [7].)

In what follows we tacitly assume that $E$ is a spectral measure on $\mathbf{R}^{n}$. The spectrum $\sigma(E)$ of $E$ is defined as

$$
\sigma(E)=\left\{\lambda \in \mathbf{R}^{n} \mid E(B(\lambda ; r)) \neq 0, \forall r>0\right\} .
$$

Let $f$ be a Borel measurable function defined on a Borel set $D \subset \mathbf{R}^{n}$. We express the integral on $D$ of $f$ with respect to the measure $(E(\cdot) u, v), u, v \in \mathcal{H}$, as $\int_{D} f(\lambda) d(E(\lambda) u, v)$. When $u=v$ we also write as $\int_{D} f(\lambda) d\|E(\lambda) u\|^{2}$.

The operator $f(E)=\int_{D} f(\lambda) d E(\lambda)$ is defined as follows:

$$
\begin{aligned}
& \mathcal{D}(f(E))=\left\{\left.u \in \mathcal{H}\left|E\left(\mathbf{R}^{n} \backslash D\right) u=0, \int_{D}\right| f(\lambda)\right|^{2} d(E(\lambda) u, u)<\infty\right\} \\
& (f(E) u, v)=\int_{D} f(\lambda) d(E(\lambda) u, v), \quad u \in \mathcal{D}(f(E)), \quad v \in \mathcal{H}
\end{aligned}
$$

Note that $D$ is not necessarily contained in $\sigma(E)$ and it should be remembered that $u \in \mathcal{D}(f(E))$ implies in particular $E\left(\mathbf{R}^{n} \backslash D\right) u=0$.

When $f$ is real valued, $f(E)$ is a selfadjoint operator in $\mathcal{H}$. In particular, letting $f_{j}(\lambda)=\lambda_{j}, \lambda=\left(\lambda_{1}, \ldots, \lambda_{n}\right)$, we put

$$
A_{j}=f_{j}(E)=\int_{\mathbf{R}^{n}} \lambda_{j} d E(\lambda)
$$


As $A_{j}$ is selfadjoint, we can write $A_{j}=\int_{-\infty}^{\infty} \lambda d E_{j}(\lambda)$ with $E_{j}(\lambda)$ being the spectral mesure on $\mathbf{R}^{1}$ associated to $A_{j}$. Then, $E_{j}$ is a commuting set of spectral measures on $\mathbf{R}^{1}$ and the relation

$$
E\left(e_{1} \times \cdots \times e_{n}\right)=E_{1}\left(e_{1}\right) \cdots E_{n}\left(e_{n}\right), \quad e_{j} \in \mathcal{B}
$$

holds. Conversely, if $E_{j}, j=1, \ldots, n$, is a commuting set of spectral measures on $\mathbf{R}^{1}$, then (1.9) determines a spectral measure on $\mathbf{R}^{n}$.

\section{$\S 1.3$. Main result}

Let $E$ be a spectral measure on $\mathbf{R}^{n}$ and let $A_{j}$ be as in (1.8). We first introduce some notations. For $u \in \bigcap_{j=1}^{n} \mathcal{D}\left(A_{j}\right)$ we put

$$
\begin{aligned}
\eta & =\eta(u ; E)=\left(\eta_{1}, \ldots, \eta_{n}\right) \in \mathbf{R}^{n}, \quad \eta_{j}=\left(A_{j} u, u\right) /\|u\|^{2}, \\
\varepsilon^{2} & =\sum_{j=1}^{n}\left\|\left(A_{j}-\eta_{j}\right) u\right\|^{2} /\|u\|^{2}, \quad \varepsilon=\varepsilon(u ; E) \geq 0 .
\end{aligned}
$$

Theorem 1.1. Let $E$ be a spectral measure on $\mathbf{R}^{n}$. Let $a \in \mathbf{R}^{n}$ and $r>0$. We assume that

$$
\operatorname{diam}(B(a ; r) \cap \sigma(E)) \leq \delta
$$

for some $\delta \geq 0$. Let $u \in \bigcap_{j=1}^{n} \mathcal{D}\left(A_{j}\right)$ and let $\eta$ and $\varepsilon$ be as in (1.10), (1.11). We assume that

$$
r^{2}-|\eta-a|^{2}-\varepsilon^{2}>0 .
$$

Then, $B(a ; r) \cap \sigma(E) \neq \emptyset$. Put

$$
B_{\delta}=B\left(\eta-\frac{\varepsilon^{2}}{r^{2}-|\eta-a|^{2}}(\eta-a) ; \frac{r \varepsilon^{2}}{r^{2}-|\eta-a|^{2}}+\delta\right) .
$$

Then we have

$$
\left\{\begin{array}{lll}
B(a ; r) \cap \sigma(E) \subset \overline{B_{\delta}}, & \text { if } \quad(\varepsilon, \delta) \neq(0,0), \\
B(a ; r) \cap \sigma(E)=\{\eta\}, & \text { if } \quad \varepsilon=\delta=0 .
\end{array}\right.
$$

Remark. 1. Assumption (1.13) implies that $\eta \in B_{0}$. It is also easy to verify that the center of $B_{\delta}$ is on the line segment joining $a$ and $\eta$ and that $\overline{B_{0}} \subset B(a ; r)$. 
2. When $\delta=0$, assumption (1.12) means that $B(a ; r) \cap \sigma(E)$ consists of at most one point and the conclusion of the theorem asserts that $B(a ; r) \cap \sigma(E)=$ $\left\{\lambda_{0}\right\}$ and that

$$
\lambda_{0} \in \bar{B}\left(\eta-\frac{\varepsilon^{2}}{r^{2}-|\eta-a|^{2}}(\eta-a) ; \frac{r \varepsilon^{2}}{r^{2}-|\eta-a|^{2}}\right) .
$$

This is exactly the generalization of Theorem A (cf. (1.4)).

3. When $\delta$ is big enough so that $\overline{B_{\delta}} \supset B(a ; r)$ (1.15) asserts nothing new. Nevertheless, we preferred not to impose any restriction on $\delta$, as the statement of the theorem is not false even though trivial.

4. When $n=2$ we may identify $\left(\lambda_{1}, \lambda_{2}\right) \in \mathbf{R}^{2}$ with $z=\lambda_{1}+i \lambda_{2} \in \mathbf{C}$. Then $E$ is a spectral measure on $\mathbf{C}$ and we have an associated normal operator $A=\int_{\mathbf{C}} z d E(z)$. As $\sigma(E)=\sigma(A)$, Theorem 1.1 is a theorem concerning the location of the spectrum of $A$.

\section{$\S 2 . \quad$ Proof of Theorem 1.1}

\section{§2.1. Preliminaries. A variational lemma}

The following simple lemma will be the basis of our argument.

Lemma 2.1. Let $E$ be a spectral measure on $\mathbf{R}^{n}$ and $f$ a real valued Borel measurable function defined on a Borel set $D \subset \mathbf{R}^{n}$. Let $w \in \mathcal{D}(f(E))$, $w \neq 0$. Then,

$$
\left\{\lambda \in D \mid f(\lambda) \leq(f(E) w, w)\|w\|^{-2}\right\} \cap \sigma(E) \neq \emptyset .
$$

Proof. For brevity put $\alpha=\inf _{\lambda \in D \cap \sigma(E)} f(\lambda)$ and $\beta=(f(E) w, w)\|w\|^{-2}$. It is obvious that

$$
(f(E) w, w)=\int_{D} f(\lambda) d(E(\lambda) w, w) \geq \alpha\|w\|^{2},
$$

so that $\beta \geq \alpha$.

If (2.1) were not true, it would mean that $f(\lambda)>\beta, \forall \lambda \in D \cap \sigma(E)$. This implies that $\alpha \geq \beta$ and hence $\alpha=\beta$ by (2.2). On the other hand, putting

$$
A_{n}=\left\{\lambda \in D \cap \sigma(E) \mid n^{-1}>f(\lambda)-\alpha \geq(n+1)^{-1}\right\}, \quad n=0,1, \ldots,
$$

where $0^{-1}=\infty$, we have $D \cap \sigma(E)=\bigcup_{n=0}^{\infty} A_{n}$, because $f(\lambda)>\beta=\alpha$, $\forall \lambda \in D \cap \sigma(E)$. Since $w \in \mathcal{D}(f(E))$ yields $E\left(\mathbf{R}^{n} \backslash D\right) w=0$, we then see that

$$
\begin{aligned}
0 & =(\beta-\alpha)\|w\|^{2}=\sum_{n=0}^{\infty} \int_{A_{n}}(f(\lambda)-\alpha) d\|E(\lambda) w\|^{2} \\
& \geq(n+1)^{-1} \int_{A_{n}} d\|E(\lambda) w\|^{2}, \quad \forall n .
\end{aligned}
$$


This would imply $E\left(A_{n}\right) w=0, \forall n$, so that $w=0$, a contradiction.

Proposition 2.1. Let $u \in \bigcap_{j=1}^{n} \mathcal{D}\left(A_{j}\right)$ and let $\eta$ and $\varepsilon$ be as in (1.10) and (1.11). Let $b \in \mathbf{R}^{n}$. Then,

$$
\begin{gathered}
\int_{\mathbf{R}^{n}}|\lambda-b|^{2} d\|E(\lambda) u\|^{2}=\left(\varepsilon^{2}+|\eta-b|^{2}\right)\|u\|^{2}, \\
\bar{B}\left(b ; \sqrt{|\eta-b|^{2}+\varepsilon^{2}}\right) \cap \sigma(E) \neq \emptyset .
\end{gathered}
$$

Proof. Noting that $\int_{\mathbf{R}^{n}}(\lambda-\eta) d\|E(\lambda) u\|^{2}=0,(2.3)$ is verified as follows:

$$
\begin{aligned}
& \int_{\mathbf{R}^{n}}|\lambda-b|^{2} d\|E(\lambda) u\|^{2}=\int_{\mathbf{R}^{n}}|\lambda-\eta+\eta-b|^{2} d\|E(\lambda) u\|^{2} \\
& \quad=\int_{\mathbf{R}^{n}}|\lambda-\eta|^{2} d\|E(\lambda) u\|^{2}+|\eta-b|^{2}\|u\|^{2}=\left(\varepsilon^{2}+|\eta-b|^{2}\right)\|u\|^{2} .
\end{aligned}
$$

For proving (2.4) take $f(\lambda)=|\lambda-b|^{2}$ in (2.1). Then, the left hand side of (2.3) is equal to $(f(E) u, u)$. Hence, $(2.1)$ with $w=u$ gives $\left\{\lambda \in \mathbf{R}^{n}|| \lambda-\left.b\right|^{2} \leq\right.$ $\left.\varepsilon^{2}+|\eta-b|^{2}\right\} \cap \sigma(E) \neq \emptyset$, which is equivalent to (2.4).

Remark. $\quad$ 1. (2.3) and (2.4), or related statements, are in [4] and [3]. The proof given above is not much different from the proofs given there.

2. For matrices relation (2.4) is proved in [9, p. 188]. In particular, with $b=\eta$ relation (2.4) says that $\bar{B}(\eta ; \varepsilon) \cap \sigma(E) \neq \emptyset$, which is a well-known fact (cf. [2, Lemme 1.26]).

\section{$\S 2.2$. Construction of balls $B(\omega)$}

It is obvious that (1.13) and (2.4) imply $B(a ; r) \cap \sigma(E) \neq \emptyset$.

The problem is not affected by a translation in $\mathbf{R}^{n}$. Furthermore, if $\varepsilon=0$, then $\eta \in \sigma(E)$ and hence (1.15) holds. Thus, in the rest of this section we shall assume without loss of generality that $a=0$ and $\varepsilon>0$.

The idea for finding $B_{0}$ of (1.14) is as follows. Fix $\omega \in \mathbf{R}^{n}$ with $|\omega|=1$ and consider a family of balls having centers on the line $\{s \omega \mid s \in \mathbf{R}\}$ and touching $S(0 ; r)$ at $r \omega$. We then apply Lemma 2.1 to this family with a special choice of $f, w$ and find a ball $B(\omega)$ such that $(\overline{B(\omega)} \backslash\{r \omega\}) \cap \sigma(E) \neq \emptyset$. This is done in this subsection. In the next subsection geometric relations between $B(\omega)$ and $B_{0}$ will be exploited.

The goal of this subsection is to prove the following proposition. 
Proposition 2.2. Let $\omega \in \mathbf{R}^{n}$ with $|\omega|=1$. Put

$$
s(\omega)=\frac{r^{2}-|\eta|^{2}-\varepsilon^{2}}{2(r \omega-\eta) \cdot \omega}=\frac{r^{2}-|\eta|^{2}-\varepsilon^{2}}{2(r-\eta \cdot \omega)} .
$$

Then $0<s(\omega)<r$. Put

$$
B(\omega)=B(s(\omega) \omega ; r-s(\omega))
$$

Then

$$
(\overline{B(\omega)} \backslash\{r \omega\}) \cap \sigma(E) \neq \emptyset
$$

Proof. Let $\omega,|\omega|=1$, be fixed and define a family $S_{\omega, s}, s \in \mathbf{R}$, consisting of spheres and a hyperplane as

$$
S_{\omega, s}= \begin{cases}S(s \omega ;|r-s|), & s \neq r \\ \{\lambda \mid(\lambda-r \omega) \cdot \omega=0\}, & s=r .\end{cases}
$$

(Though $\omega$ is fixed throughout this subsection, it is included in the subscript of $S_{\omega, s}$ for clarity.) Geometry is that, for $0<s<r, S_{\omega, s}$ is contained in $B(0 ; r) \cup\{r \omega\}$ and is inscribed to $S(0 ; r)$ at the point $r \omega$. (For the definition of "inscribed" see the beginning of $\S 2.3$.) For $s=0 S_{\omega, 0}=S(0 ; r)$. We omit similar description for other cases.

Let $D=\mathbf{R}^{n} \backslash\{r \omega\}$ and define the mapping $\Phi_{r \omega}: D \rightarrow \mathbf{R}^{n}$ as

$$
\Phi_{r \omega}(\lambda)=r^{2} \frac{\lambda-r \omega}{|\lambda-r \omega|^{2}}+r \omega, \quad \lambda \in D .
$$

$\Phi_{r \omega}$ is a mapping of inversion with respect to the center $r \omega$ scaled so that each point on the sphere $S(r \omega ; r)$ remains invariant. We then define the mapping $f: D \rightarrow \mathbf{R}^{n}$ appearing in Lemma 2.1 as

$$
f(\lambda)=\Phi_{r \omega} \cdot \omega=r^{2} \frac{(\lambda-r \omega) \cdot \omega}{|\lambda-r \omega|^{2}}+r, \quad \lambda \in D .
$$

Let $S_{\omega, s}^{\prime}=S_{\omega, s} \cap D=S_{\omega, s} \backslash\{r \omega\}$. Each of $S_{\omega, s}^{\prime}$ is a level surface of $f(\lambda)$. More precisely, we shall see that

$$
f(\lambda)=f_{s}, \quad \lambda \in S_{\omega, s}^{\prime}, \quad \text { where } f_{s}= \begin{cases}r+\frac{r^{2}}{2(s-r)}, & s \neq r, \\ r, & s=r .\end{cases}
$$

This is obvious for $s=r$. For $s \neq r$ a generic point of $S_{\omega, s}$ is expressed as $\lambda=s \omega+|s-r| \kappa,|\kappa|=1$. Then $\lambda-r \omega=(s-r) \omega+|s-r| \kappa$ and hence 
$(\lambda-r \omega) \cdot \omega=s-r+|s-r| \kappa \cdot \omega,|\lambda-r \omega|^{2}=2(s-r)(s-r+|s-r| \kappa \cdot \omega)$.

for $s \neq r$ follows from these and (2.8) at once.

Let now $u, \eta, \varepsilon$ be as in the theorem and put

$$
w=\int_{\mathbf{R}^{n}}|\lambda-r \omega| d E(\lambda) u .
$$

Assumption $u \in \bigcap_{j=1}^{n} \mathcal{D}\left(A_{j}\right)$ implies $\int_{\mathbf{R}^{n}}|\lambda-r \omega|^{2} d\|E(\lambda) u\|^{2}<\infty$, so that $w$ is well-defined. We also see by (2.10) that $E(\{r \omega\}) w=0$. Since $|f(\lambda)| \leq$ $r^{2}|\lambda-r \omega|^{-1}+r$, we have $w \in \mathcal{D}(f(E))$ and $(f(E) w, w)$ is computed as follows:

$$
\begin{aligned}
(f(E) w, w) & =\int_{\mathbf{R}^{n}} f(\lambda) d\|E(\lambda) w\|^{2} \\
& =\int_{\mathbf{R}^{n}} r^{2}((\lambda-r \omega) \cdot \omega) d\|E(\lambda) u\|^{2}+r\|w\|^{2} \\
& =r^{2}((\eta-r \omega) \cdot \omega)\|u\|^{2}+r\|w\|^{2} .
\end{aligned}
$$

On the other hand, (2.3) with $b=r \omega$ gives

$$
\|w\|^{2}=\int_{\mathbf{R}^{n}}|\lambda-r \omega|^{2} d\|E(\lambda) u\|^{2}=\left(\varepsilon^{2}+|\eta-r \omega|^{2}\right)\|u\|^{2} .
$$

Thus we obtain

$$
(f(E) w, w)\|w\|^{-2}=r-\frac{r^{2}(r \omega-\eta) \cdot \omega}{\varepsilon^{2}+|\eta-r \omega|^{2}} .
$$

Since $a=0$, assumption (1.13) gives $\varepsilon^{2}<r^{2}-|\eta|^{2}$. As this implies $\eta \in B(0 ; r)$, we have $(r \omega-\eta) \cdot \omega>0$. Using these two relations we see that

$$
\frac{r^{2}(r \omega-\eta) \cdot \omega}{\varepsilon^{2}+|\eta-r \omega|^{2}}>\frac{r^{2}(r-\eta \cdot \omega)}{r^{2}-|\eta|^{2}+|\eta-r \omega|^{2}}=\frac{r}{2} .
$$

This shows that the right hand side of (2.11) is less than $r / 2$. From the shape of $f_{s}$ and the fact that $f_{0}=r / 2$, it then follows that the equation

$$
f_{s}=(f(E) w, w)\|w\|^{-2}
$$

has a unique solution $s=s(\omega)$ and $0<s(\omega)<r$. By (2.9) and (2.11) we see that this solution is exctly $s(\omega)$ given by $(2.5)$. That $0<s(\omega)<r$ was proved above, or it is easy to verify it directly from (2.5). As $s(\omega)<r, S_{\omega, s(\omega)}=$ $S(s(\omega) \omega ; r-s(\omega))$. Hence, $B(\omega)$ defined by $(2.6)$ is the ball whose boundary is $S_{\omega, s(\omega)}$. Noting that $f_{s} \leq f_{s(\omega)}$ if and only if $s(\omega) \leq s<r$, we finally see that

$$
\left\{\lambda \in D \mid f(\lambda) \leq(f(E) w, w)\|w\|^{-2}\right\}=\bigcup_{s(\omega) \leq s<r} S_{\omega, s}^{\prime}=\overline{B(\omega)} \backslash\{r \omega\} .
$$

Then, (2.7) follows from (2.1). 
Remark. 1. Balls $B(\omega)$ can also be obtained by applying Proposition 2.1 to the family of balls $B(s \omega ; r-s \omega)$. This method is closer to the original method of [4], [3] and manipulations to reach $B(\omega)$ may be a little shorter. But then the conclusion we can obtain is $\overline{B(\omega)} \cap \sigma(E) \neq \emptyset$ and there remains the possibility that $\overline{B(\omega)} \cap \sigma(E)=\{r \omega\}$. In order to bypass this difficulty, we would need to introduce a limit argument.

2. When $n=2$, there corresponds to a spectral measure $E$ a normal operator $A$. Then, the method of using the mapping of inversion $\Phi$ is closely related (almost equivalent) to considering the Rayleigh bound of $\operatorname{Re}\{\bar{\omega}((A-$ $\left.\left.r \omega)^{-1} w, w\right)\right\}$, where $\omega \in \mathbf{C}$ with $|\omega|=1$. We should note that, for selfadjoint operators, Lehman ([5]), Maehly ([6]), and more recently Zimmermann and Mertins ([10]) used Rayleigh-Ritz procedure applied to the resolvent to obtain bounds for arbitrary eigenvalues.

\section{§2.3. Relation between $B(\omega)$ and $B_{0}$}

Consider two spheres $S_{1}=S\left(a_{1} ; r_{1}\right), S_{2}=S\left(a_{2} ; r_{2}\right)$ with $r_{1}<r_{2}$. If the relation

$$
\left|a_{2}-a_{1}\right|+r_{1}=r_{2}
$$

holds, then $S_{1} \cap S_{2}=\{p\}$, where $p=a_{j}+r_{j}\left|a_{1}-a_{2}\right|^{-1}\left(a_{1}-a_{2}\right), j=1,2$, and $S_{1} \subset B\left(a_{2} ; r_{2}\right) \cup\{p\}$. In this case we say that $S_{1}$ is inscribed to $S_{2}$ at $p$. $S_{1}$ and $S_{2}$ have a common tangent hyperplane at $\{p\}$ given by $\left\{\lambda \mid(\lambda-p) \cdot\left(a_{1}-a_{2}\right)=0\right\}$. (This is illustrated in Figure 1 for $n=2$ in the situation to be described in Proposition 2.3.)

Returning to the situation of $\S 2.2$, let $S_{0}$ and $S(\omega)$ be the boundary sphere of $B_{0}$ and $B(\omega)$, respectively. For the brevity of notation put

$$
Q=r^{2}-|\eta|^{2}-\varepsilon^{2}, \quad \zeta=r \omega-\eta
$$

By (1.14) with $a=0$ and (2.5) $S_{0}$ and $S(\omega)$ are then expressed as

$$
\begin{aligned}
& S_{0}=S(\xi ; \rho) ; \quad \xi=\frac{Q}{Q+\varepsilon^{2}} \eta, \quad \rho=\frac{r \varepsilon^{2}}{Q+\varepsilon^{2}}, \\
& S(\omega)=S(s(\omega) \omega ; r-s(\omega)), \quad s(\omega)=\frac{Q}{2 \zeta \cdot \omega} .
\end{aligned}
$$

Proposition 2.3. For each $\omega$ with $|\omega|=1$ the relations

$$
r-s(\omega)>\rho, \quad|s(\omega) \omega-\xi|+\rho=r-s(\omega)
$$




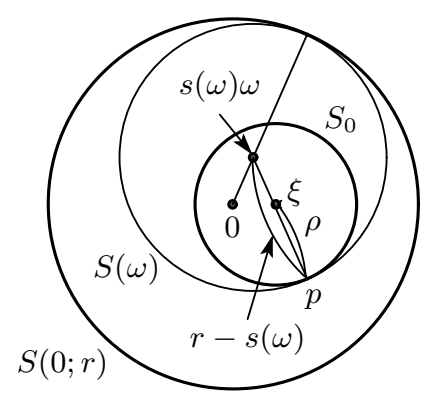

Figure 1. $S_{0}$ is inscribed to $S(\omega) \quad(n=2)$

hold, so that $S_{0}$ is inscribed to $S(\omega)$ (Figure 1). Conversely, for each $p \in S_{0}$ there exists $\omega_{1}$ with $\left|\omega_{1}\right|=1$ such that $S_{0}$ is inscribed to $S\left(\omega_{1}\right)$ at $p$.

Proof. The proof of (2.17) is by mechanical computations. To prove the first relation we first note that $2 r \zeta \cdot \omega=|\zeta|^{2}+r^{2}-|\zeta-r \omega|^{2}=|\zeta|^{2}+r^{2}-|\eta|^{2}=$ $|\zeta|^{2}+Q+\varepsilon^{2}$. Then by (2.16) we obtain

$$
r-s(\omega)=\frac{r\left(|\zeta|^{2}+\varepsilon^{2}\right)}{|\zeta|^{2}+Q+\varepsilon^{2}}>\frac{r \varepsilon^{2}}{Q+\varepsilon^{2}}=\rho .
$$

Since $r-s(\omega)-\rho>0$ as proved, the second relation of (2.17) is equivalent to $|s(\omega) \omega-\xi|^{2}=(r-s(\omega)-\rho)^{2}$, which in turn is rewritten as

$$
|\xi|^{2}-2 s(\omega) \xi \cdot \omega=(r-\rho)^{2}-2(r-\rho) s(\omega) .
$$

By (2.15) we obtain $r-\rho=r Q\left(Q+\varepsilon^{2}\right)^{-1}$. Use this, (2.15), and (2.16) in (2.19) and multiply the resulting equation by $Q^{-2}\left(Q+\varepsilon^{2}\right)^{2} \zeta \cdot \omega$. Then, we see that (2.19) is equivalent to

$$
|\eta|^{2} \zeta \cdot \omega-\left(Q+\varepsilon^{2}\right) \eta \cdot \omega=r^{2} \zeta \cdot \omega-r\left(Q+\varepsilon^{2}\right) .
$$

As $r^{2}-|\eta|^{2}=Q+\varepsilon^{2}$, equation $(2.20)$ is equivalent to $(\zeta+\eta) \cdot \omega=r$, which is true because $\zeta=r \omega-\eta$. Thus, the second relation of (2.17) is verified.

To prove the converse statement let $p \in S_{0}$ and consider the family of spheres $S_{t}=S\left(\xi+t|\xi-p|^{-1}(\xi-p) ; \rho+t\right), t \geq 0$. (Note that $S_{0}$ by this definition coincides with the original $S_{0}$.) For $t>0 S_{0}$ is inscribed to $S_{t}$ at $p$. As confirmed by continuity argument there exists $t_{1}>0$ and $p_{1} \in S(0 ; r)$ such that $S_{t_{1}}$ is inscribed to $S(0 ; r)$ at $p_{1}$. Let $\omega_{1}=\left|p_{1}\right|^{-1} p_{1}$. Then it is clear that $S_{t_{1}}=S\left(\omega_{1}\right)$ and that this $\omega_{1}$ is what we wanted to find. 
Remark. The proof given above uses a conjectured shape of $B_{0}$ and is not a heuristic proof. Alternatively, $S_{0}$ can be obtained by computing the enveloping surface of the family of spheres $\{S(\omega)|| \omega \mid=1\}$. (The amount of computation is comparable.) We avoided this heuristic proof, because it requires some delicate argument after the equation of the enveloping surface was computed by a formal calculus.

\section{$\S 2.4$. Completion of the proof}

Proof is by contradiction. It is illustrated in Figure 2 for $n=2$.

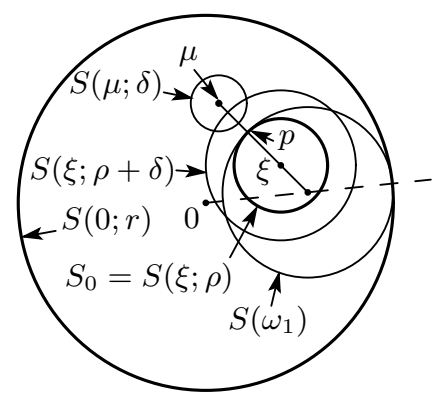

Broken line indicates direction $\omega_{1}$

Figure 2. Proof by contradiction

Let $B_{0}=B(\xi ; \rho)$ and $S_{0}=S(\xi ; \rho)$ be as before. We know that $B(0 ; r) \cup$ $\sigma(E) \neq \emptyset$ holds. Suppose now that (1.15) (with $a=0$ and $\varepsilon>0$ ) were false. Then, there would exist a $\mu \in B(0 ; r) \cap \sigma(E)$ such that

$$
|\mu-\xi|>\rho+\delta
$$

By (1.12) we also have

$$
B(0 ; r) \cap \sigma(E) \subset \bar{B}(\mu ; \delta) .
$$

Let $p$ be the intersection of the segment $\overline{\xi \mu}$ joining $\xi$ and $\mu$ with the sphere $S_{0}$. Apply the converse part of Proposition 2.3 to this $p$ and find $\omega_{1}$ as in the proposition. Then, by $(2.21)$ and $(2.22)$ we see that $\overline{B\left(\omega_{1}\right)}$ and $B(0 ; r) \cap \sigma(E)$ are separated by the plane tangent to $B\left(\omega_{1}\right)$ at $p$. This contradict the fact that $B\left(\omega_{1}\right)$ satisfies (2.7). Hence, (1.15) must hold.

The proof of Theorem 1.1 is now complete. 
Remark. By a similar argument we can prove that $\overline{B_{0}}=\bigcap_{|\omega|=1} \bar{B}(\omega)$.

\section{$\S 3 . \quad$ An Invariance Property}

\section{§3.1. Mapping of inversion}

Estimate (1.15) has a certain invariance property when the spectral measure is transformed by a mapping of inversion in $\mathbf{R}^{n}$. The purpose of this section is to present a theorem (Theorem 3.1 given below) showing this type of invariance. What is crucial is the fact that an inversion maps a ball either to a ball, to the outside of a ball, or to a half space.

As in Theorem 1.1 let $E$ be a spectral measure in $\mathbf{R}^{n}$ and let $a \in \mathbf{R}^{n}$, $r>0$. We restrict ourselves to the case that the center of the inversion $b$ is neither in $\bar{B}(a ; r)$ nor in $\sigma(E)$. We may and shall take $b=0$ without loss of generality. Thus, throughout this section we assume that

$$
0 \notin \bar{B}(a ; r), \quad 0 \notin \sigma(E)
$$

and consider the mapping $\Psi: \mathbf{R}^{n} \backslash\{0\} \longrightarrow \mathbf{R}^{n}$ defined by

$$
\Psi(\lambda)=p^{2} \frac{\lambda}{|\lambda|^{2}}, \quad \lambda \in \mathbf{R}^{n} \backslash\{0\},
$$

where $p>0$ is a fixed positive number. $\Psi$ is the inversion in $\mathbf{R}^{n}$ with respect to the origin which leaves the sphere $S(0 ; p)$ invariant. We could take $p=1$ without loss of generality, but here we prefer to keep $p$, as $p$ has the "dimension" of the length and it is convenient to make it visible in various formulas.

It is evident that $\Psi$ is one-to-one and maps $\mathbf{R}^{n} \backslash\{0\}$ onto itself and that $\Psi^{-1}=\Psi$.

The following proposition is elementary and can be verified immediately.

Proposition 3.1. Under assumption (3.1) we have

$$
\Psi(B(a ; r))=B(\widehat{a} ; \widehat{r}), \quad \widehat{a}=\frac{p^{2}}{|a|^{2}-r^{2}} a, \quad \widehat{r}=\frac{p^{2} r}{|a|^{2}-r^{2}} .
$$

Note that the first relation of (3.1) is equivalent to $r<|a|$ so that the denominators in (3.3) are positive. We also have $\widehat{r}<|\widehat{a}|$ so that $0 \notin B(\widehat{a} ; \widehat{r})$. 


\section{§3.2. Statement of results}

Throuout the rest of this section we consider the situation described in Theorem 1.1 with additional assumption (3.1). Furthermore we restrict ourselves to the case that $\delta=0$. Notations $E$, $u$, etc. will be used without further comments.

We define the transformed spectral measure $\widehat{E}: \mathcal{B} \rightarrow \mathcal{P}$ as follows:

$$
\widehat{E}(e)=E\left(\Psi^{-1}(e)\right)=E(\Psi(e)), \quad \forall e \in \mathcal{B} .
$$

It is easy to verify that $\widehat{E}$ is a spectral measure. (Note that the assumption $0 \notin \sigma(E)$ yields $\widehat{E}\left(\mathbf{R}^{n}\right)=I$.) The spectral mapping theorem holds so that $\sigma(\widehat{E})=\Psi(\sigma(E)) \cup \gamma$, where $\gamma=\{0\}$ if $\sigma(E)$ is not bounded and $\gamma=\emptyset$ if $\sigma(E)$ is bounded. Furthermore, $0 \notin \sigma(E)$ implies that an open ball with the center 0 is contained in $\sigma\left(\mathbf{R}^{n}\right) \backslash \sigma(E)$, which in turn implies that $\sigma(\widehat{E})$ is a compact set.

Let $A_{j}$ be as defined by (1.8) and put

$$
A=\left(A_{1}^{2}+\cdots+A_{n}^{2}\right)^{1 / 2}=\int_{\mathbf{R}^{n}}\left(\lambda_{1}^{2}+\cdots+\lambda_{n}^{2}\right)^{1 / 2} d E(\lambda) .
$$

In parallel to $A_{j}$ the operator $\widehat{A}_{j}$ is defined by the first line of the following (3.5) and is then computed as follows by means of the change of measure argument. (Note that, as $0 \notin \sigma(E), A^{-1}$ exists and bounded.)

$$
\begin{aligned}
\widehat{A}_{j} & =\int_{\mathbf{R}^{n}} \mu_{j} d \widehat{E}(\mu) \\
& =\int_{\mathbf{R}^{n}} \Psi(\lambda)_{j} d E(\lambda)=p^{2} \int_{\mathbf{R}^{n}} \frac{\lambda_{j}}{|\lambda|^{2}} d E(\lambda)=p^{2} A_{j} A^{-2} .
\end{aligned}
$$

In particcular, $\widehat{A}$ defined similarly to (3.4) satisfies $\widehat{A}=p^{2} A^{-1}$.

Noting that the assumption $u \in \bigcap_{j=1}^{n} \mathcal{D}\left(A_{j}\right)$ implies $u \in \mathcal{D}(A)$, we choose our transformed trial vector $\widehat{u}$ as

$$
\widehat{u}=A u
$$

and construct $\widehat{\eta}$ and $\widehat{\varepsilon}$ by (1.10) and (1.11) with ^ everywhere. Proposition 3.2 to be given below shows that $\widehat{\eta}$ and $\widehat{\varepsilon}$ can be computed from $\eta$ and $\varepsilon$ and it is not necessary to compute $(\widehat{A} \widehat{u}, \widehat{u})$. In this respect $\widehat{u}$ defined by (3.6) may be the most probable candidate for a transformed trial vector.

Starting from $\{E, B(a ; r), u\}$ we constructed the ball $B_{0}$ of (1.14). $B_{0}$ gives an estimate for $\sigma(E)$. Likewise, starting from $\{\widehat{E}, B(\widehat{a} ; \widehat{r}), \widehat{u}\}$, we can construct 
$\widehat{B}_{0}$ in a similar way. $\widehat{B}_{0}$ gives an estimate for $\sigma(\widehat{E})$. The question is whether $\Psi^{-1}\left(\widehat{B}_{0}\right)$ gives an estimate better than (or at least different from) the original estimate by $B_{0}$. The following theorem asserts that it is not the case and in fact $\Psi^{-1}\left(\widehat{B}_{0}\right)=B_{0}$, or $\widehat{B}_{0}=\Psi\left(B_{0}\right)$.

Theorem 3.1. Let $Q^{\prime}=r^{2}-|\eta-a|^{2}-\varepsilon^{2}$ and $\widehat{Q}^{\prime}=\widehat{r}^{2}-|\widehat{\eta}-\widehat{a}|^{2}-\widehat{\varepsilon}^{2}$. Then

(i) $\widehat{Q}^{\prime}>0$ if and only if $Q^{\prime}>0$. In other words, assumption (1.13) holds for the triplet $\{\widehat{E}, B(\widehat{a} ; \widehat{r}), \widehat{u}\}$, if and only if it holds for $\{E, B(a ; r), u\}$;

(ii) if $Q^{\prime}>0$, then $\widehat{B}_{0}=\Psi\left(B_{0}\right)$.

Remark. Assumption (3.1) implies $a \neq 0$, which is opposite to the simplification $a=0$ used in $\S 2.2$ and $\S 2.3$. In the notation $Q^{\prime}$ a prime is added to distinguish it from $Q$ of (2.14) used in the case of $a=0$.

\section{§3.3. $\quad$ Proof of Theorem 3.1}

The proof will be done by a series of rather mechanical manipulations.

Proposition 3.2. $\widehat{\eta}, \widehat{\varepsilon}$, and $\widehat{Q}^{\prime}$ are related to $\eta, \varepsilon$, and $Q^{\prime}$ as follows.

$$
\begin{gathered}
\widehat{\eta}=\frac{p^{2}}{|\eta|^{2}+\varepsilon^{2}} \eta, \quad \widehat{\varepsilon}^{2}=\frac{p^{4}}{\left(|\eta|^{2}+\varepsilon^{2}\right)^{2}} \varepsilon^{2}, \\
\widehat{Q}^{\prime}=\frac{p^{4} Q^{\prime}}{\left(|a|^{2}-r^{2}\right)\left(|\eta|^{2}+\varepsilon^{2}\right)} .
\end{gathered}
$$

Proof. By putting $b=0$ in (2.3) we obtain

$$
\|A u\|^{2}=\left(\varepsilon^{2}+|\eta|^{2}\right)\|u\|^{2} .
$$

Recalling (3.5) the first equality of (3.7) is verified as follows.

$$
\widehat{\eta}_{j}=\frac{\left(\widehat{A}_{j} \widehat{u}, \widehat{u}\right)}{\|\widehat{u}\|^{2}}=\frac{\left(p^{2} A_{j} A^{-2} A u, A u\right)}{\|A u\|^{2}}=\frac{p^{2}\left(A_{j} u, u\right)}{\|A u\|^{2}}=\frac{p^{2}}{|\eta|^{2}+\varepsilon^{2}} \eta_{j}
$$

Relation (3.9) holds with ${ }^{\wedge}$ everywhere. Using it in a reverse way together with $\widehat{A}=p^{2} A^{-1}$ and (3.10), we see that

$$
\begin{aligned}
\widehat{\varepsilon}^{2} & =\frac{\|\widehat{A} \widehat{u}\|^{2}}{\|\widehat{u}\|^{2}}-|\widehat{\eta}|^{2}=\frac{p^{4}\|u\|^{2}}{\|A u\|^{2}}-\frac{p^{4}}{\left(|\eta|^{2}+\varepsilon^{2}\right)^{2}}|\eta|^{2} \\
& =\frac{p^{4}}{|\eta|^{2}+\varepsilon^{2}}-\frac{p^{4}}{\left(|\eta|^{2}+\varepsilon^{2}\right)^{2}}|\eta|^{2}=\frac{p^{4} \varepsilon^{2}}{\left(|\eta|^{2}+\varepsilon^{2}\right)^{2}} .
\end{aligned}
$$


For proving relation (3.8) we insert expressions (3.3) and (3.7) into the right hand side of $\widehat{Q}^{\prime}=\widehat{r}^{2}-|\widehat{\eta}-\widehat{a}|^{2}-\widehat{\varepsilon}^{2}$ and obtain

$$
\begin{aligned}
\widehat{Q}^{\prime} & =p^{4}\left(|a|^{2}-r^{2}\right)^{-2}\left(|\eta|^{2}+\varepsilon^{2}\right)^{-2} R, \\
R & =r^{2}\left(|\eta|^{2}+\varepsilon^{2}\right)^{2}-\left|\left(|a|^{2}-r^{2}\right) \eta-\left(|\eta|^{2}+\varepsilon^{2}\right) a\right|^{2}-\varepsilon^{2}\left(|a|^{2}-r^{2}\right)^{2} \\
& =\left(|a|^{2}-r^{2}\right)\left(|\eta|^{2}+\varepsilon^{2}\right)\left(-|\eta|^{2}-\varepsilon^{2}-\left(|a|^{2}-r^{2}\right)+2 \eta \cdot a\right) \\
& =\left(|a|^{2}-r^{2}\right)\left(|\eta|^{2}+\varepsilon^{2}\right) Q^{\prime} .
\end{aligned}
$$

Hence, (3.8) follows.

Proof of Theorem 3.1. (i) is clear by (3.8) because $|a|^{2}-r^{2}>0$ by assumption (3.1).

Proof of (ii). We know by Theorem 1.1 that $B_{0}=B\left(\xi^{\prime} ; \rho^{\prime}\right)$ and $\widehat{B}_{0}=$ $B\left(\widehat{\xi}^{\prime} ; \widehat{\rho}^{\prime}\right)$, where

$$
\xi^{\prime}=\frac{Q^{\prime}}{Q^{\prime}+\varepsilon^{2}} \eta+\frac{\varepsilon^{2}}{Q^{\prime}+\varepsilon^{2}} a, \quad \rho^{\prime}=\frac{r \varepsilon^{2}}{Q^{\prime}+\varepsilon^{2}}
$$

and similarly for $\widehat{\xi}^{\prime}$ and $\widehat{\rho}^{\prime}$ with - everywhere. We first note that $0 \notin B(a ; r)$ and $B_{0} \subset B(a ; r)$ imply $0 \notin B_{0}$. In particular, $\left|\xi^{\prime}\right|^{2}-\rho^{\prime 2}>0$. For simplicity we put $h^{2}=\left|\xi^{\prime}\right|^{2}-\rho^{\prime 2}, h>0$. Then, by Proposition 3.1 we have $\Psi\left(B_{0}\right)=$ $B\left(p^{2} h^{-2} \xi^{\prime} ; p^{2} h^{-2} \rho^{\prime}\right)$. Thus, it suffices to prove the following two relations:

$$
\begin{gathered}
\frac{p^{2}}{h^{2}} \xi^{\prime}=\frac{\widehat{Q}^{\prime}}{\widehat{Q}^{\prime}+\widehat{\varepsilon}^{2}} \widehat{\eta}+\frac{\widehat{\varepsilon}^{2}}{\widehat{Q}^{\prime}+\widehat{\varepsilon}^{2}} \widehat{a}, \\
\frac{p^{2} \rho^{\prime}}{h^{2}}=\frac{\widehat{r} \widehat{\varepsilon}^{2}}{\widehat{Q}^{\prime}+\widehat{\varepsilon}^{2}} .
\end{gathered}
$$

Proof of (3.12). As vectors in $\mathbf{R}^{n}$ both sides are linear combinations of $\eta$ and $a$. By equating coefficients of $\eta$ and $a$, we find that it suffices to prove

$$
\begin{aligned}
& \frac{Q^{\prime}}{h^{2}\left(Q^{\prime}+\varepsilon^{2}\right)}=\frac{\widehat{Q}^{\prime}}{\left(\widehat{Q}^{\prime}+\widehat{\varepsilon}^{2}\right)\left(|\eta|^{2}+\varepsilon^{2}\right)}, \\
& \frac{\varepsilon^{2}}{h^{2}\left(Q^{\prime}+\varepsilon^{2}\right)}=\frac{\widehat{\varepsilon}^{2}}{\left(\widehat{Q}^{\prime}+\widehat{\varepsilon}^{2}\right)\left(|a|^{2}-r^{2}\right)} .
\end{aligned}
$$

Proof of (3.14). On the right hand side we first see by (3.7) and (3.8) that

$$
\widehat{Q}^{\prime}+\widehat{\varepsilon}^{2}=\frac{p^{4} T}{\left(|a|^{2}-r^{2}\right)\left(|\eta|^{2}+\varepsilon^{2}\right)^{2}},
$$




$$
\begin{aligned}
T & =Q^{\prime}\left(|\eta|^{2}+\varepsilon^{2}\right)+\left(|a|^{2}-r^{2}\right) \varepsilon^{2} \\
& =-\varepsilon^{4}+2\left(\eta \cdot a-|\eta|^{2}\right) \varepsilon^{2}+\left(r^{2}-|\eta-a|^{2}\right)|\eta|^{2} .
\end{aligned}
$$

Using (3.8) again we see that the right hand side of (3.14) is equal to $Q^{\prime} T^{-1}$. On the left hand side we see that

$$
h^{2}=\left|\xi^{\prime}\right|^{2}-\rho^{\prime 2}=\frac{\left(|a|^{2}-r^{2}\right) \varepsilon^{4}+2 Q^{\prime} \eta \cdot a \varepsilon^{2}+Q^{\prime 2}|\eta|^{2}}{\left(Q^{\prime}+\varepsilon^{2}\right)^{2}} .
$$

By a straightforward computation the numerator on the right hand side of this relation is seen to be equal to $\left(r^{2}-|\eta-a|^{2}\right) T=\left(Q^{\prime}+\varepsilon^{2}\right) T$. This and (3.16) show that the left hand side of (3.14) is also equal to $Q^{\prime} T^{-1}$. Thus (3.14) is proved.

Proof of (3.15). Multiply equation (3.14) by $\varepsilon^{2} / Q^{\prime}$. The left hand side of the resulting equation is equal to that of (3.15). That the right hand side is equal to that of (3.15) is readily seen by (3.7) and (3.8). Thus, (3.15) is verified.

This completes the proof of (3.12).

Proof of (3.13). Multiply both sides of (3.15) by $p^{2} r$. It is easily seen that each side of the resulting equation is equal to the corresponding side of (3.13).

The proof of Theorem 3.1 is now complete.

\section{References}

[1] M. S. Birman and M. Z. Solomjak, Spectral theory of self-adjoint operators in Hilbert space, Translated from the Russian by S. Khrushchev and V. Peller, Math. Appl. (Soviet Series), D. Reidel, Dordrecht, 1987.

[2] F. Chatelin, Spectral approximation of linear operators, Academic Press, New York, 1983.

[3] E. M. Harrell, II, Generalizations of Temple's inequality, Proc. Amer. Math. Soc. 69 (1978), no. 2, 271-276.

[4] T. Kato, On the upper and lower bounds of eigenvalues, J. Phys. Soc. Japan 4 (1949), 334-339.

[5] N. J. Lehmann, Beiträge zur numerischen Lösung linearer Eigenwertprobleme. I-II, Z. Angew. Math. Mech. 29 (1949), 341-356. ibid., 30 (1950), 1-16.

[6] H. J. Maehly, Ein neues Variationsverfahren zur genäherten Berechnung der Eigenwerte hermitescher Operatoren. (German) Helvetica Phys. Acta 25 (1952), 547-568.

[7] Yu. S. Samollenko, Spectral theory of families of selfadjoint operators, Translated from the Russian by E. V. Tisjachnij, Kluwer Acad. Publ., Dordrecht, 1991.

[8] G. Temple, The theory of Rayleigh's principle as applied to continuous systems, Proc. Royal Soc. London Ser. A 119 (1928), 276-293.

[9] J. H. Wilkinson, The algebraic eigenvalue problem, Clarendon Press, Oxford, 1965.

[10] S. Zimmermann and U. Mertins, Variational bounds to eigenvalues of self-adjoint eigenvalue problems with arbitrary spectrum, Z. Anal. Anwendungen 14 (1995), no. 2, 327345. 